\title{
Platelet-Dense Granules Worsen Pre-Infection Thrombocytopenia during Gram-Negative Pneumonia-Derived Sepsis
}

\author{
Theodora A.M. Claushuis ${ }^{a} \quad$ Alex F. de Vos ${ }^{a}$ Joris J.T.H. Roelofs ${ }^{b}$ \\ Onno J. de Boer ${ }^{b}$ Cornelis van 't Veer ${ }^{a}$ Tom van der Poll ${ }^{a, c}$ \\ ${ }^{a}$ Center for Experimental and Molecular Medicine, Academic Medical Center, University of Amsterdam, Amsterdam, \\ The Netherlands; ${ }^{\mathrm{b}}$ Department of Pathology, Academic Medical Center, Amsterdam, The Netherlands; ${ }^{\mathrm{C}}$ Division of \\ Infectious Diseases, Academic Medical Center, University of Amsterdam, Amsterdam, The Netherlands
}

\section{Keywords}

Animal models · Bacterial infection · Host defense $\cdot$ Platelets · Sepsis

\begin{abstract}
Platelet-dense $(\delta)$ granules are important for platelet function. Platelets contribute to host defense and vascular integrity during pneumonia and sepsis, and $\delta$ granule products, including adenosine diphosphate (ADP), can influence inflammatory responses. We therefore aimed to study the role of platelet $\delta$ granules in the host response during sepsis. Hermansky-Pudlak syndrome ( $\mathrm{Hps}$ ) 3 coa mice (with reduced $\delta$ granule content), mice treated with the platelet ADP receptor inhibitor clopidogrel, and appropriate control mice were infected with the human sepsis pathogen Klebsiella pneumoniae via the airways to induce pneumonia and sepsis. In order to override potential redundancy in platelet functions, we also studied $\mathrm{Hps} 3 \mathrm{coa}$ and control mice with moderate antibody-induced thrombocytopenia (10\%) prior to infection. We found that sepsis-induced thrombocytopenia tended to be less severe in Hps3coa mice, and was significantly ameliorated in Hps3coa mice with low pre-infection platelet counts. Bacterial growth was similar in Hps3coa and control
\end{abstract}

\begin{tabular}{ll}
\hline KARGER & ( 2018 The Author(s) \\
& Published by S. Karger AG, Basel Oparger \\
E-Mail karger@karger.com & This article is licensed under the Creative Commons Attribution- \\
www.karger.com/jin & NonCommercial-NoDerivatives 4.0 International License (CC BY- \\
NC-ND) (http://www.karger.com/Services/OpenAccessLicense). \\
Usage and distribution for commercial purposes as well as any dis- \\
tribution of modified material requires written permission.
\end{tabular}

mice in the presence of normal platelet counts prior to infection, but lower in the lungs of Hps3coa mice with low preinfection platelet counts. Hps3coa mice had unaltered lung pathology and distant organ injury during pneumosepsis, irrespective of pre-infection platelet counts; lung bleeding did not differ between Hps3coa and control mice. Clopidogrel did not influence any host response parameter. These data suggest that platelet $\delta$ granules can play a detrimental role in pneumosepsis by aggravating thrombocytopenia and impairing local antibacterial defense, but that these unfavorable effects only become apparent in the presence of low platelet counts. (c) 2018 The Author(s)

Published by S. Karger AG, Basel

\section{Introduction}

Platelets contain several granules crucial for their function, including dense $(\delta)$ granules [1]. $\delta$ granules are lysosome-related granules, with an average of $3-8 \delta$ granules per platelet and a size of around $150 \mathrm{~nm}$ in diameter [2]. $\delta$ granules have a varied content, including ionic species such as calcium, magnesium and polyphosphate, membrane proteins including CD63 and lysosome-asso-

Dr. Theodora Agnes Maria Claushuis

Center for Experimental and Molecular Medicine, Academic Medical Center Meibergdreef 9, Room F0-117

NL-1105 AZ Amsterdam (The Netherlands)

E-Mail t.a.claushuis@amc.uva.nl 
ciated membrane protein-2, nucleotides adenosine triphosphate, and adenosine diphosphate (ADP), as well as the bioactive amines serotonin and histamine, and transporter protein multidrug resistance protein (MRP4) [3, $4]$. The $\delta$ granule component ADP is crucial for platelet activation by mediating a positive feedback loop after platelet activation [5]. Moreover, the $\delta$ granule components ADP, serotonin, and polyphosphates can directly influence inflammatory responses $[1,5-8]$.

Apart from a crucial role in hemostasis, platelets are important players in host defense during infection $[9,10]$. Recent investigations have shown that platelets aid in host defense in multiple ways, including P-selectin [11], glycoprotein (GP)VI [12], and GPIb [13, 14]. Platelets can crucially modulate immunity during infection and inflammation, influencing, for example, leukocyte recruitment, cytokine production, and neutrophil extracellular trap formation [15-18].

Moreover, platelets are crucial for protection against infection-induced bleeding $[12,19]$. The role of $\delta$ platelet granules in host defense or vascular integrity during sepsis is unknown.

We therefore aimed to determine the role of platelet $\delta$ granules in the host response during pneumonia-derived sepsis. To investigate this, mice with decreased $\delta$ granule content, mice with reduced ADP signaling, and appropriate control animals were infected with the Gram-negative bacterium Klebsiella pneumoniae - a frequent cause of sepsis in humans $[20,21]$ - to produce a gradually evolving localized infection which disseminates to distant body sites.

\section{Methods}

\section{Animals}

Specific pathogen-free C57Bl/6 mice (Charles River, Lyon, France), Hermansky-Pudlak syndrome (Hps3) ${ }^{c o a}$ mice, and littermates (Jackson, Bar Harbor, ME, USA) were used for infection experiments. $H p s 3^{c o a}$ mice have abnormal $\delta$ granules with a decreased platelet granule content, but in contrast to other HPS mice have unaffected lysosomal granules [22-24]. All experiments were conducted with gender-matched mice between 8 and 12 weeks of age. The Institutional Animal Care and Use Committee of the Academic Medical Center approved all experiments.

\section{Experimental Study Design}

Pneumonia and sepsis was induced by intranasal inoculation with K. pneumoniae serotype 2 (ATCC 43816, Rockville, MD, USA; 10,000 colony-forming units, CFU, in $50 \mu \mathrm{L}$ of isotonic saline), as previously described $[12,19]$. Mice were euthanized 12 or $40 \mathrm{~h}$ after the induction of pneumonia ( $n=8$ per group); noninfected mice were sacrificed simultaneously ( $n=4$ per group). In some experiments, $H p s 3^{c o a}$ mice and littermates were intravenously injected with platelet-depleting antibody (polyclonal antimouse-GPIba, $0.2 \mu \mathrm{g} / \mathrm{g}$ ) or control immunoglobulin G (both Emfret Analytics, Eibelstadt, Germany) [19]. C57Bl/6 wild-type mice were treated by oral gavage with clopidogrel $(30 \mu \mathrm{g} / \mathrm{g}$; A pothecon, Barneveld, The Netherlands) or PBS control once daily, from 3 days before infection until the moment of sacrifice. Bacterial quantification and storage of lung, blood, liver, and spleen were performed as previously described [19]. Tail bleeding times were determined after 3 days of clopidogrel treatment by standardized cut from the tail, after which bleeding was timed in PBS at $37^{\circ} \mathrm{C}$.

\section{Flow Cytometry}

Flow cytometry was conducted with FACSCalibur (Becton Dickinson, Franklin Lakes, NJ, USA). Murine platelet counts were measured using hamster anti-mouse-CD61 mAb (BioLegend, San Diego, CA, USA). Platelet activation was measured using rat antimouse CD62P (BD Biosciences) and rat anti-mouse CD63 (Thermo Fisher Scientific, Waltham, MA, USA). Murine platelet-leukocyte complex formation was determined by using rat anti-mouse CD11b mAb (BD Biosciences) and rat anti-mouse Ly-6G mAb (both BD Biosciences) or rat anti-mouse-CD115 mAb (eBioscience) in combination with hamster anti-mouse-CD61 mAb.

\section{Protein Measurements}

Interleukin 6 (IL-6), tumor necrosis factor- $\alpha(\mathrm{TNF} \alpha)$, and chemokine (C-C motif) ligand 2 (CCL2) were determined with a cytometric beads array multiplex assay (BD Biosciences). E-selectin, myeloperoxidase (MPO), C-X-C motif ligand 2 (CXCL2) and CXCL4 (all R\&D Systems, Minneapolis, MN, USA), thrombin-antithrombin complexes (TATc; Bio-Connect, Huissen, The Netherlands), and serotonin (Enzo Life Sciences, Zandhoven, Belgium) were measured by ELISA. Aminotransferase (AST), alanine aminotransferase (ALT), and lactate dehydrogenase (LDH) were measured using a c702 Roche Diagnostics (Roche Diagnostics BV, Almere, The Netherlands). Hemoglobin concentrations were measured in 50 -fold diluted lung homogenates by light absorption at $410 \mathrm{~nm}$ by a NanoDrop spectrophotometer (Thermo Fisher Scientific).

\section{Ex vivo Stimulations}

Citrated blood from control or Hps $3^{c o a}$ mice was stimulated with protease-activated receptor-4-activating peptide (PAR4AP; 0.1 and $0.4 \mathrm{mg} / \mathrm{mL}$; American Peptide Company, Sunnyvale, CA, USA) for $30 \mathrm{~min}$ at room temperature or with UV-radiated $K$. pneumoniae $\left(10^{6} \mathrm{CFU}\right)$ for $6 \mathrm{~h}$ at $37^{\circ}$. Platelet activation was assessed by FACS and blood was spun at 3,000 $\mathrm{g}$ to collect plasma. Citrated blood from control or $\mathrm{Hps} 3^{\mathrm{coa}}$ mice was also incubated for $2 \mathrm{~h}$ with viable $K$. pneumoniae $\left(10^{6} \mathrm{CFU} / \mathrm{mL}\right)$, after which growth was assessed by plating bacteria on blood agar plates.

\section{Pathology}

The paraffin-embedded left lung lobe was cut into $4-\mu \mathrm{m}$ sections and stained with hematoxylin and eosin. Slides were coded and scored by a pathologist blinded for group identity as previously described [19]. In short, the following parameters were scored in lungs from 0 (absent) to 4 (most severe): interstitial inflammation, endothelialitis, bronchitis, edema, pleuritis, bleeding, and the presence of thrombi; in the liver the following parameters were scored from 0 to 4: inflammation, presence of thrombi, and 
presence of necrosis. The total histopathology score was calculated as the sum of the scores of all individual parameters. To determine neutrophil influx in the lung, sections were stained with antimouse Ly-6G mAb (BD Pharmingen, San Diego, CA, USA) as previously described [19]. After staining, expression was quantified by digital image analysis: slides were scanned using an Olympus dotSlide virtual slide microscope (Olympus, Tokyo, Japan) to generate TIFF images of the full tissue section. Ly- $6 \mathrm{G}$ positivity was measured using Image J (US National Institutes of Health, Bethesda, $\mathrm{MD}$, USA); the amount of positivity was expressed as the percentage of the total lung surface area.

\section{Statistical Analysis}

Data are expressed as scatter dot plots with lines showing the median. Comparisons between groups at multiple time points were first performed using a (nonparametric) Kruskal-Wallis test; groups at individual time points were tested using the MannWhitney U test only when significant differences were present. If only one time point was present for the two-group comparison, a Mann-Whitney U test was used directly. Analyses were made using GraphPad Prism version 5.01 (GraphPad Software, San Diego, CA, USA). $p$ values $<0.05$ were considered statistically significant.

\section{Results}

Hps $3^{\text {coa }}$ Mice Have Abnormal Platelet $\delta$ Granule Content with Unaltered Leukocyte Granularity

In line with previous studies showing reduced $\delta$ granule content in $H p s 3^{c o a}$ mice [22-24], granularity was reduced in platelets from $H p s 3^{c o a}$ mice (as assessed by side-scatter; Fig. 1a, $p<0.001$ vs. controls). Platelet counts were similar between groups (Fig. 1b). In accordance, after stimulation with low-dose PAR4AP, the expression of CD63 (derived from $\delta$ and lysosomal granules [25]) was reduced (Fig. 1c, $p<0.05$ vs. control). Pselectin expression was also decreased in $\mathrm{Hps} 3^{\text {coa }}$ platelets after low-dose PAR4AP stimulation (Fig. $1 \mathrm{~d}, p<0.05$ vs. control), pointing at absent positive feedback by ADP release due to lack of $\delta$ granules [1]. Stimulation with a higher dose of PAR4AP resulted in higher P-selectin expression, which was not different between groups, with CD63 expression still decreased in $H p s 3^{\text {coa }}$ platelets (Fig. 1e-f, $p<0.05$ vs. control). Moreover, plasma levels of $\delta$ granule product serotonin were strongly reduced in $H p s 3^{\text {coa }}$ mice (Fig. 1g, $p<0.05$ vs. controls), while levels of the alpha granule product CXCL4 were not (Fig. 1h) after stimulation with high-dose PAR4AP. Neutrophil and monocyte counts and granularity were unaffected (Fig. $1 \mathrm{i}-\mathrm{j}$ ). These data show that $\mathrm{Hps}^{\text {coa }}$ mice have reduced $\delta$ granule content, whereas other granules or leukocytes are unaffected.
Mice with Reduced Platelet $\delta$ Granule Secretion Show a Trend towards Reduced Thrombocytopenia during

\section{Pneumonia and Sepsis}

During platelet activation, release of ADP from $\delta$ granules results in a positive feedback loop, which is important for platelet activation [1]. Inhibition of ADP signaling has been shown to inhibit platelet-leukocyte complex formation [5]. We previously showed that sepsis induces platelet activation, platelet-leukocyte complex formation, and thrombocytopenia [12]. Moreover, human studies have shown an immunomodulatory effect of ADP inhibition by clopidogrel in a human endotoxemia model [26]. Therefore, we assessed the role of $\delta$ granules and ADP herein by using $H p s 3^{c o a}$ mice and wild-type mice treated with clopidogrel (an irreversible inhibitor of the P2Y12 ADP platelet receptor [27]). Akin to before infection, $H p s 3^{c o a}$ mice also showed decreased platelet granularity during pneumosepsis induced by infection with $K$. pneumoniae via the airways (online suppl. Fig. S1; see www. karger.com/doi/10.1159/000494147 for all online suppl. material). Mice treated with clopidogrel showed increased tail bleeding times, confirming the biological activity of this compound (online suppl. Fig. S2). Sepsis induced thrombocytopenia during later stages of the infection $\left(40 \mathrm{~h}\right.$ ), which tended to be less severe in $H p s 3^{c o a}$ mice (Fig. 2a, $p=0.07$ ). Clopidogrel treatment did not affect platelet counts during sepsis (Fig. 2f). Platelet activation as measured by CXCL4 plasma levels was reduced in $H p s 3^{c o a}$ mice $40 \mathrm{~h}$ after infection (Fig. $2 \mathrm{~b}, p<0.005$ vs. controls), but not in clopidogrel-treated mice; P-selectin expression and platelet-leukocyte complex formation were similar for all groups during pneumosepsis (Fig. 2c$e, h-j)$. These data suggest that platelet $\delta$ granules might contribute to sepsis-induced platelet activation and thrombocytopenia.

\section{Low Pre-Infection Platelet Counts Reveal a Role for Platelet $\delta$ Granules in Sepsis-Induced Thrombocytopenia}

We previously reported that the protective effects of platelets during pneumosepsis are dependent on platelet counts, wherein a small rise in platelet numbers (from $<5$ to $5-13 \times 10^{9} / \mathrm{L}$ ) already improved host defense and largely prevented hemorrhage [19]. Therefore, we wished to assess the importance of $\delta$ granules in the more stressed setting of reduced pre-infection platelet counts, wherein the remaining platelets would need to function optimally after infection. Thus, we partially depleted platelets in $H p s 3^{c o a}$ and control mice by anti-GPIba treatment prior to infection to yield platelet counts of around $10 \%$ of 


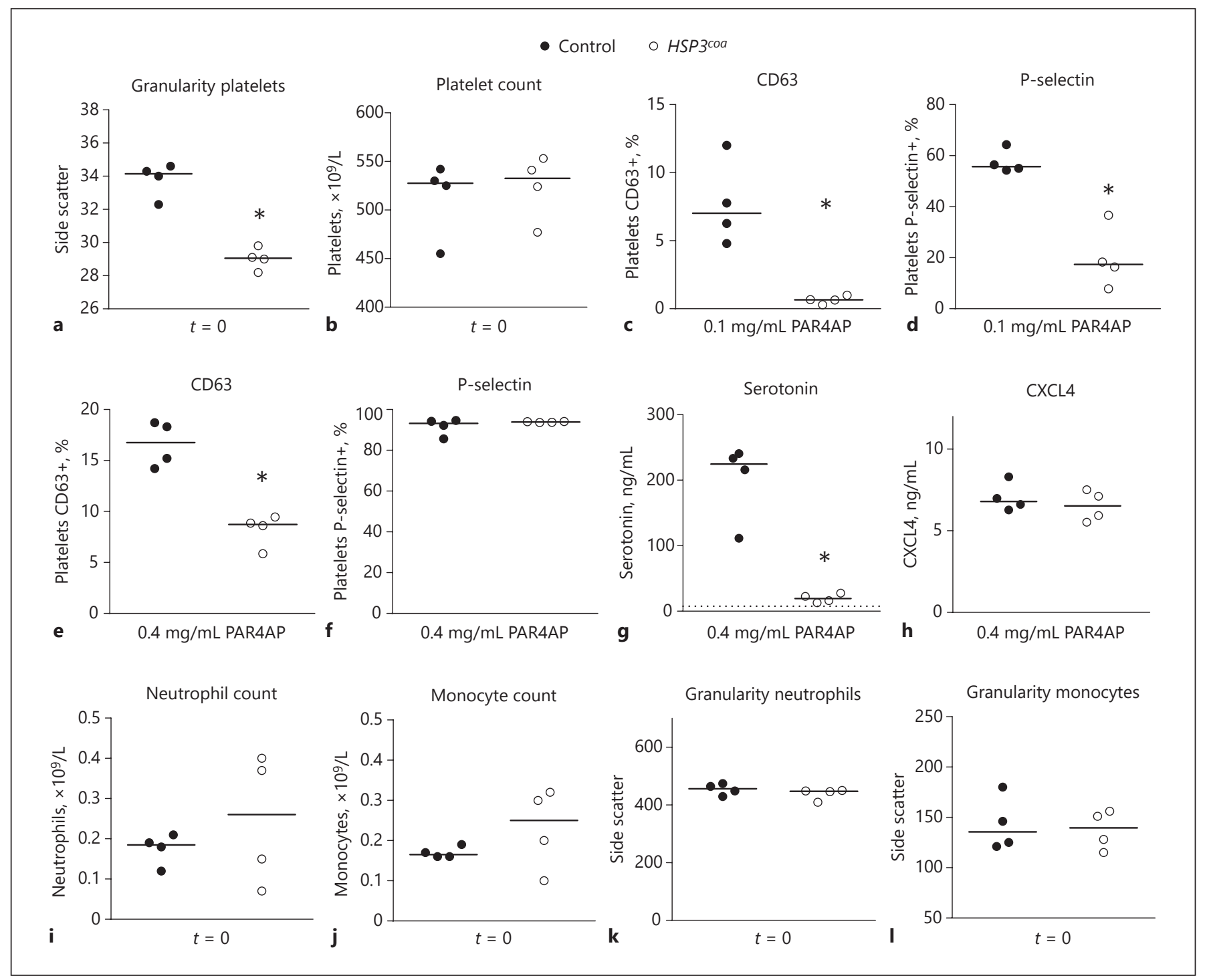

Fig. 1. Hps $3^{c o a}$ mice have abnormal platelet $\delta$ granules with unaltered leukocyte granularity. a Platelet side-scatter. b Platelet counts. Platelet CD63 (c, e) and P-selectin $(\mathbf{d}, \mathbf{f})$ expression in $H p s 3^{c o a}$ and control blood stimulated with $0.1 \mathrm{mg} / \mathrm{mL}(\mathbf{c}, \mathbf{d})$ or $0.4 \mathrm{mg} / \mathrm{mL}(\mathbf{e}, \mathbf{f})$ of PAR4AP. Plasma levels of serotonin $(\mathbf{g})$ and
CXCL4 (h) after stimulation with $0.4 \mathrm{mg} / \mathrm{mL}$ of PAR4AP. Neutrophil (i) and monocyte (j) counts. Neutrophil (k) and monocyte (I) side scatters. Data are represented as scatter dot plots of 4 mice per group. Lines show the median. ${ }^{*} p<0.05$ versus controls. normal $[19,28]$; anti-GPIba reduced platelet counts to a similar extent in $H p s 3^{c o a}$ and control mice (online suppl. Fig. S3). In mice with pre-infection thrombocytopenia, sepsis induced a further decline in platelet counts, which was significantly less in $H p s 3^{c o a}$ than in control mice (Fig. 2k, $p<0.005$ vs. controls). Plasma CXCL4 levels, platelet $\mathrm{P}$-selectin expression, and platelet-leukocyte complex formation did not differ between groups during pneumosepsis (Fig. 2l-o). Together these results suggest that normal pre-infection platelet counts in part mask the role of $\delta$ granules in sepsis-induced thrombocytopenia.

\section{Platelet $\delta$ Granules Impair Host Defense during}

Pneumosepsis in Mice with Low Pre-Infection Platelet

Counts

Considering the role of platelets in antibacterial defense in this model of pneumosepsis [19], we next studied the role of platelet $\delta$ granules herein. To this end we 


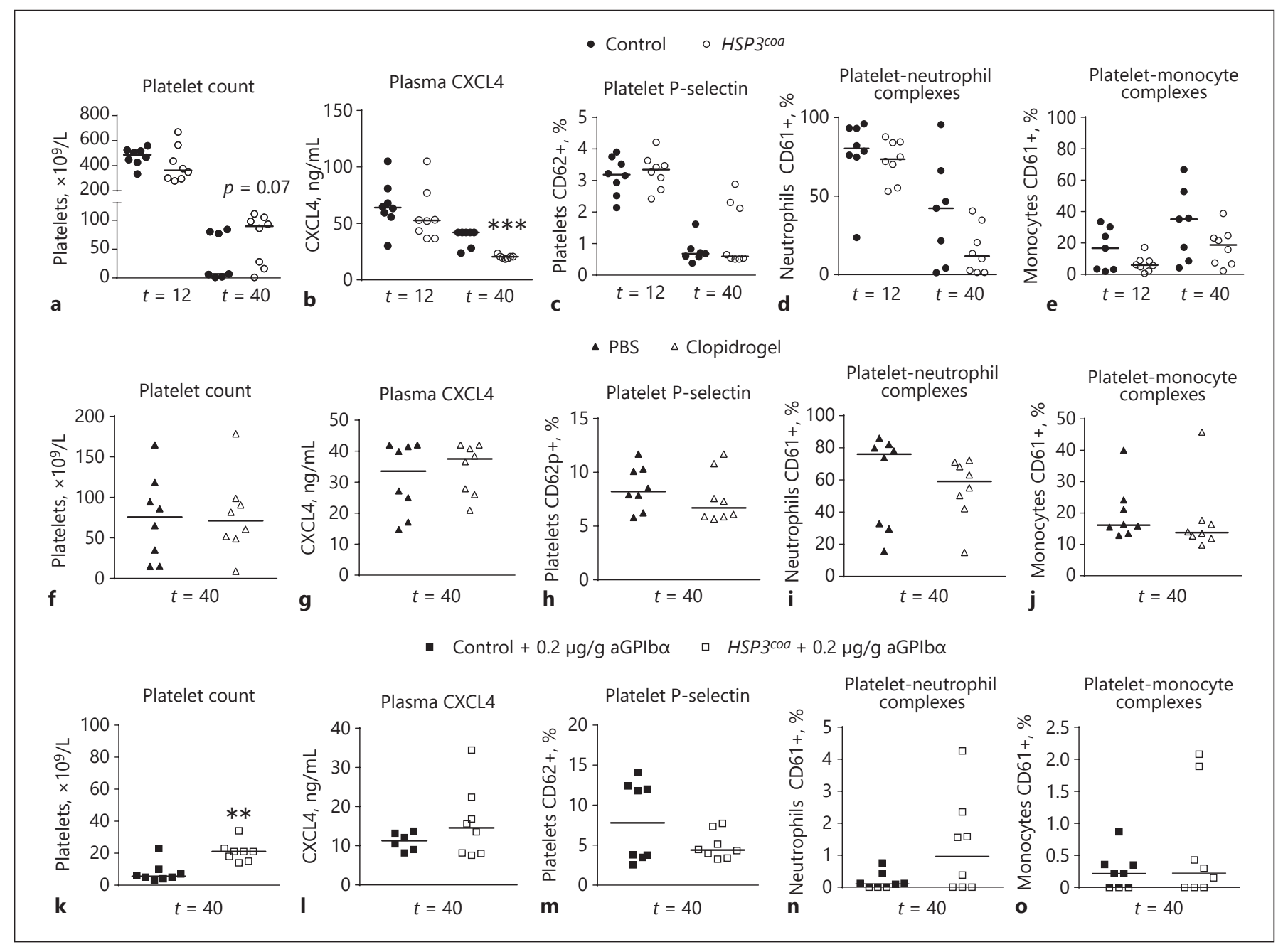

Fig. 2. Platelet responses during pneumosepsis. a-e Control or $H p s 3^{\text {coa }}$ mice were infected with K. pneumoniae via the airways and sacrificed 12 or $40 \mathrm{~h}$ after infection. Platelet counts (a), plasma CXCL4 levels (b), platelet P-selectin expression (c), platelet-neutrophil (d) and platelet-monocyte (e) complexes. $\mathbf{f}-\mathbf{j}$ Mice treated with PBS or clopidogrel were infected with K. pneumoniae via the airways and sacrificed $40 \mathrm{~h}$ after infection. Platelet counts (f), plasma CXCL4 levels (g), platelet P-selectin expression (h), platelet- neutrophil (i) and platelet-monocyte (j) complexes. k-o Control or $\mathrm{Hps} 3^{\mathrm{coa}}$ mice were injected with anti-GPIba antibody to deplete platelet counts to approximately $10 \%$, infected with K. pneumoniae via the airways, and sacrificed $40 \mathrm{~h}$ after infection. Platelet counts (k), plasma CXCL4 levels (I), platelet P-selectin expression $(\mathbf{m})$, platelet-neutrophil (n) and platelet-monocyte (o) complexes. Data are represented as scatter dot plots of 8 mice per group. Lines show the median. ${ }^{* *} p<0.005,{ }^{* * *} p<0.001$ versus controls. quantified bacterial loads in lungs and distant organs of $H p s 3^{\text {coa }}$ and control mice infected with $K$. pneumoniae. $\delta$ granule deficiency did not impair host defense, with similar bacterial loads in $\mathrm{Hps}^{\text {coa }}$ and control mice in all body sites (Fig. 3a-d). Also, in vitro K. pneumoniae growth in blood from $\mathrm{Hps} 3^{c o a}$ and control mice was similar (online suppl. Fig. S4). Inhibition of ADP has been reported to influence immune responses in humans [5]. To assess the effect of specific inhibition of ADP signaling, we also compared bacterial loads in clopidogrel-treated mice and controls, which were similar between groups (Fig. 3e-h). Considering the platelet count-dependent role of platelets in antibacterial defense in this model [19], we furthermore assessed bacterial loads in $H p s 3^{c o a}$ and control mice with low (approx. 10\%) pre-infection platelet counts. In this stressed setting bacterial loads were lower in the lungs of $H p s 3^{c o a}$ mice (Fig. 3i, $p<0.005$ vs. controls with approx. $10 \%$ platelet counts); bacterial loads in blood and distant organs were unaffected (Fig. $3 j-1)$. These data suggest that platelet $\delta$ granules can harm antibacterial defense at the 


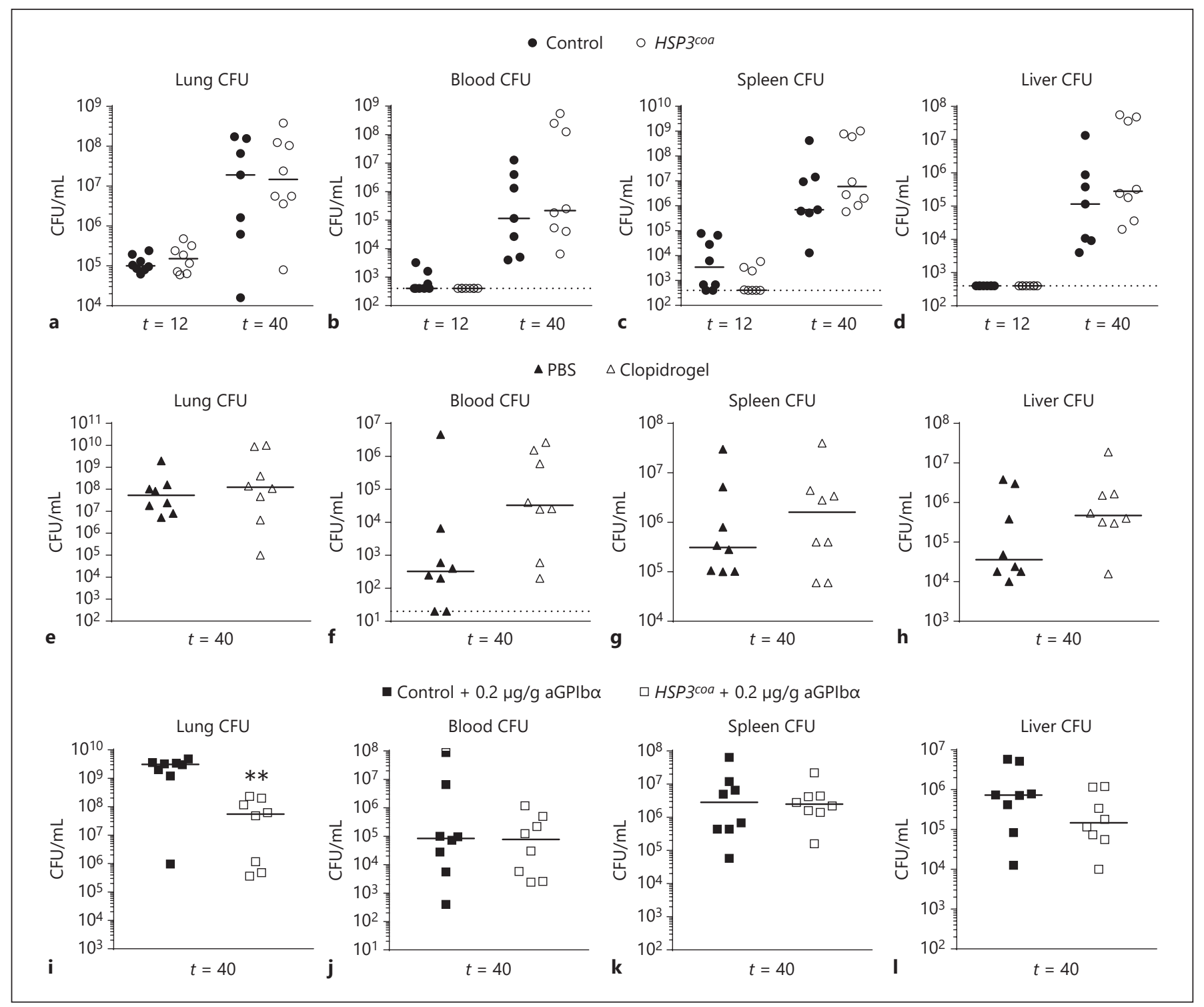

Fig. 3. Bacterial growth and dissemination during pneumosepsis. a-d Control or Hps $3^{\text {coa }}$ mice were infected with K. pneumoniae via the airways and sacrificed 12 or $40 \mathrm{~h}$ after infection. e-h Mice treated with PBS or clopidogrel were infected with K. pneumoniae via the airways, and sacrificed $40 \mathrm{~h}$ after infection. i-I Control or $H p s 3^{c o a}$ mice were injected with anti-GPIba antibody to deplete platelet counts to approximately $10 \%$, infected with K. pneumoniae via the airways, and sacrificed $40 \mathrm{~h}$ after infection. Data are the number of CFU in the body site indicated and presented as scatter dot plots of 8 mice per group. Lines show the median. ${ }^{* *} p<0.005$ versus controls. primary site of infection during pneumosepsis, but that this detrimental role is overridden in hosts with normal pre-infection platelet counts.

\section{Platelet $\delta$ Granules Do Not Influence Lung \\ Inflammation during Pneumosepsis}

$\delta$ granules have content that can directly influence immunity, including leukocyte recruitment and cytokine production $[1,5]$. We therefore assessed the effect of $\delta$ granule deficiency and inhibition of ADP signaling on cell influx and inflammation during pneumosepsis. Neutrophil recruitment, neutrophil degranulation (as measured by MPO), and lung and plasma cytokine (IL-6, $\mathrm{TNF} \alpha$ ) and chemokine (CCL2) levels were similar between $H p s 3^{c o a}$ and control mice (Fig. 4a-f; online suppl. Fig. S5). Likewise, the extent and characteristics of lung 


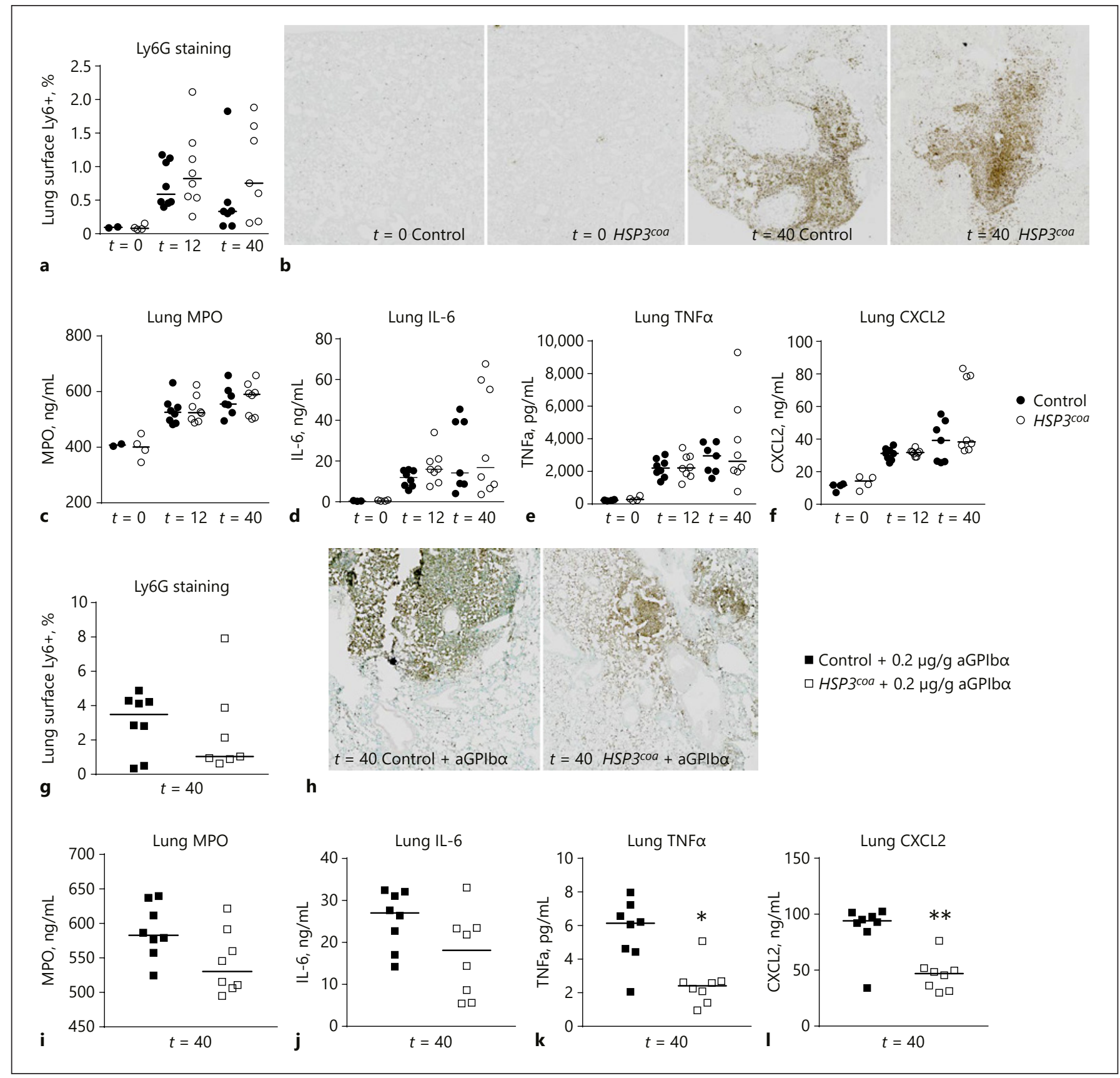

Fig. 4. Neutrophil recruitment and lung cytokine levels during pneumosepsis. a-f Control or Hps $3^{\text {coa }}$ mice were infected with $K$. pneumoniae via the airways and sacrificed 12 or $40 \mathrm{~h}$ after infection. Ly6G staining lung sections (a), representative photos (original magnification $\times 10$; b). Lung MPO (c), IL-6 (d), TNFa (e), and CXCL2 (f) levels. g-I Control or Hps $3^{c o a}$ mice were injected with anti-GPIba antibody to deplete platelet counts to approximately
$10 \%$, infected with $K$. pneumoniae via the airways, and sacrificed $40 \mathrm{~h}$ after infection. Ly6G staining lung sections (g), representative photos (original magnification $\times 10$; h). Lung MPO (i), IL-6 (j), TNFa (k), and CXCL2 (I) levels. Data are represented as scatter dot plots of 8 mice per group. Lines show the median. ${ }^{*} p<0.05$, ** $p<0.005$ versus controls. 

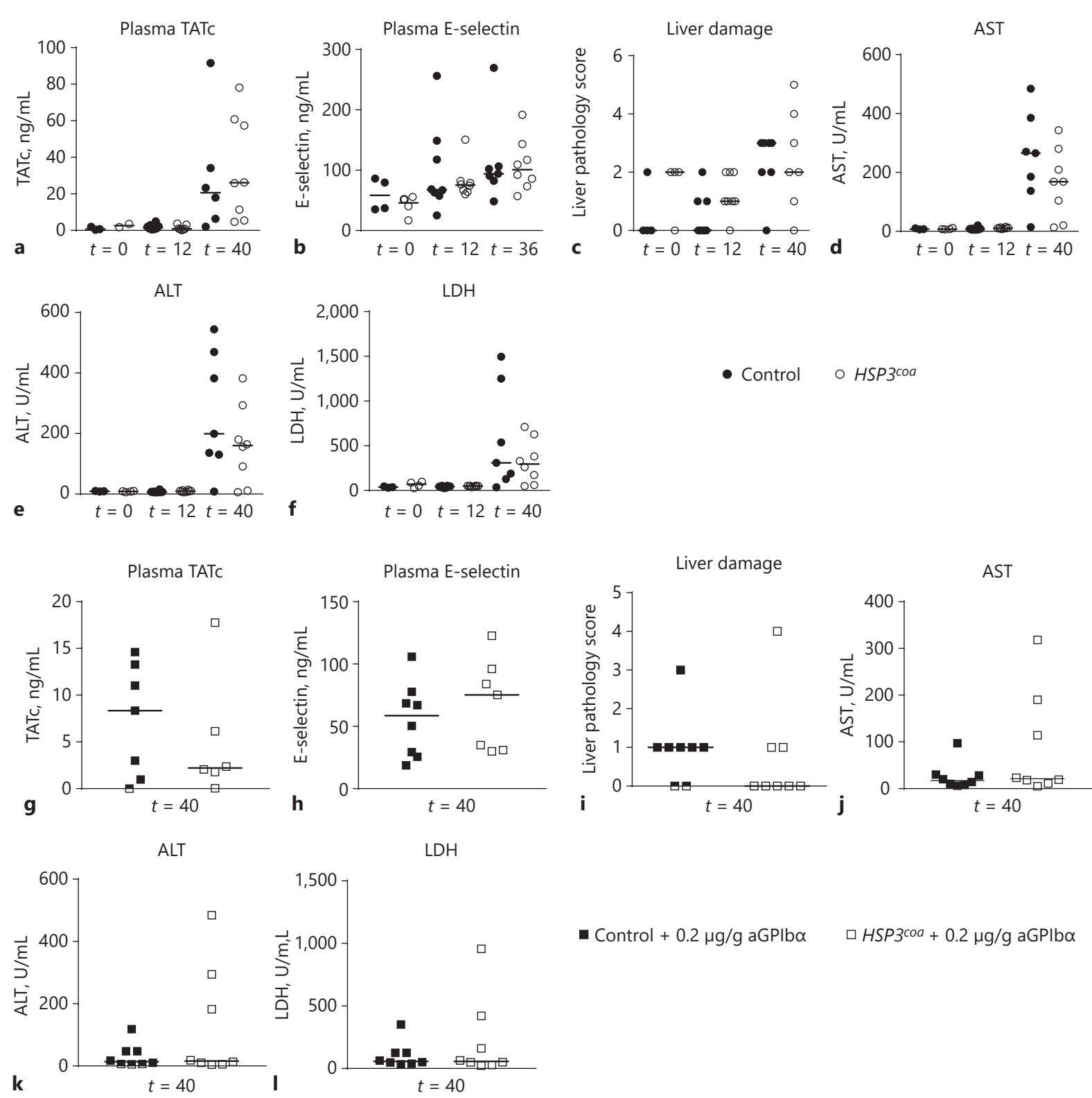

- Control + $0.2 \mu \mathrm{g} / \mathrm{g}$ aGPIb $\alpha \quad \square$ HSP3coa $+0.2 \mu \mathrm{g} / \mathrm{g}$ aGPIb $\alpha$

Fig. 5. Coagulation, endothelial cell activation, and distant organ injury. a-f Control or Hps $3^{c o a}$ mice were infected with $K$. pneumoniae via the airways and sacrificed 12 or $40 \mathrm{~h}$ after infection. Plasma TATc (a) and E-selectin levels (b). c Liver pathology score. Plasma AST (d), ALT (e), and LDH (f) levels. g-I Control or $H p s 3^{c o a}$ mice were injected with anti-GPIba antibody to deplete

pathology, as determined by the scoring system described in the Methods section, did not differ between groups (online suppl. Fig. S6). Moreover, whole blood from $H p s 3^{c o a}$ and control mice stimulated with UV-radiated

Platelet-Dense Granules in Pneumonia and Sepsis platelet counts to approximately $10 \%$, infected with K. pneumoniae via the airways, and sacrificed $40 \mathrm{~h}$ after infection. Plasma TATc (g) and E-selectin levels (h). i Liver pathology score. Plasma AST (j), $\operatorname{ALT}(\mathbf{k})$, and LDH (I) levels. Data are represented as scatter dot plots of 8 mice per group. Lines show the median. Differences between groups were not significant.
Klebsiella did not show differences in cytokine levels or neutrophil degranulation (online suppl. Fig. S7).

Furthermore, for clopidogrel-treated mice, no differences were found in the aforementioned readouts for in- 
flammation except for higher lung IL-6 levels compared to controls (online suppl. Fig. S5, S6, and S8, $p<0.05$ vs. control). In line with decreased bacterial loads in the lungs of $H p s 3^{c o a}$ mice with approximately $10 \%$ pre-infection platelet counts, lung TNFa and CXCL2 levels were lower in platelet-depleted $H p s 3^{c o a}$ mice compared to controls (Fig. $4 \mathrm{k}-\mathrm{l}, p<0.05$ vs. controls). Furthermore, plasma IL-6 levels were lower in $H p s 3^{c o a}$ mice than in controls in the setting of low pre-infection platelet counts (online suppl. Fig. S5). Neutrophil influx into lung tissue (Fig. 4gh) and lung pathology (online suppl. Fig. S6) did not differ between groups. These data suggest that platelet $\delta$ granules do not have a major impact on important inflammatory responses during Gram-negative pneumosepsis.

\section{Platelet $\delta$ Granules Do Not Influence Activation} of Coagulation and the Vascular Endothelium, or Distant Organ Damage during Pneumosepsis

Platelets can contribute to activation of coagulation and endothelial cells, and their interplay has been implicated in sepsis-induced organ damage $[19,29]$. The $\delta$ granule component polyphosphate can directly enhance coagulation via the intrinsic pathway $[1,7]$. To assess the effect of platelet $\delta$ granules on coagulation during pneumosepsis, we determined plasma TATc levels, which were similar between $\mathrm{Hps} 3^{\text {coa }}$ mice (with or without preinfection thrombocytopenia) and clopidogrel-treated mice, and their respective controls (Fig. 5a, g; online suppl. Fig. S9). Likewise, endothelial activation (measured by soluble E-selectin plasma levels) was not different between groups (Fig. 5b, h; online suppl. Fig. S9). To assess the effects of platelet $\delta$ granules on distant organ damage during pneumosepsis, we quantified liver pathology and measured the plasma concentrations of AST, ALT (reflecting hepatocellular injury), and LDH (reflecting cell injury in general). All aforementioned parameters were similar in $\mathrm{Hps}^{\text {coa }}{ }^{\text {mice }}$ (with or without pre-infection thrombocytopenia) and clopidogrel-treated mice and their respective controls (Fig. 5c-f, i-l; online suppl. Fig. S9). These data suggest that platelet $\delta$ granules do not influence activation of coagulation or the endothelium, and do not contribute to distant organ damage during Gram-negative pneumosepsis.

\section{Platelet $\delta$ Granules Are Not Involved in Vascular Integrity during Pneumonia and Sepsis}

Platelets safeguard lung vascular integrity during pneumosepsis $[12,19]$. To determine the role of platelet $\delta$ granules herein, we assessed the extent of lung bleeding by histopathology and measured lung hemoglobin levels $40 \mathrm{~h}$ after infection. In mice with normal pre-infection platelet counts (either $H p s 3^{c o a}$ mice, clopidogrel-treated mice, or their respective controls) no significant lung bleeding was detected (Fig. 6a-b, d-e). In $H p s 3^{c o a}$ mice with approximately $10 \%$ pre-infection platelet counts modest lung hemorrhage was detected, but to a similar extent as littermate controls with pre-infection thrombocytopenia (Fig. $6 \mathrm{c}, \mathrm{f}-\mathrm{g}$ ). These data argue against a role for platelet $\delta$ granules in the prevention of bleeding during infection.

\section{Discussion}

Platelets are important players in host defense during infection $[9,10]$ and platelet granules are crucial for their function [1]. Here we used $H p s 3^{c o a}$ mice to determine the role of platelet $\delta$ granules in the host response during Gram-negative pneumonia-derived sepsis, and complemented these studies by inhibiting P2Y12, the platelet receptor for ADP. ADP (a major product of $\delta$ granules) induces a positive platelet-activating feedback loop, and also influences platelet-leukocyte complex formation and cytokine levels during infection and inflammation $[5,26$, $30]$. Our main finding is that platelet $\delta$ granules can play a detrimental role in pneumosepsis by aggravating thrombocytopenia and in this way impairing local antibacterial defense, but that these unfavorable effects only become apparent in the presence of low platelet counts (Fig. 7). In this setting, the remaining platelets would need to function optimally, revealing the effects of $\delta$ granules on platelet functions in a more stressed situation. Potential redundancy in different pathways of platelet activation might therefore be reduced.

Platelet $\delta$ granules have content that can directly influence immunity $[1,5]$, including serotonin $[6,8]$, polyphosphate [7], and ADP [26]. Serotonin can enhance leukocyte rolling, adhesion, and extravasation during lipopolysaccharide-induced inflammation, which in the case of endotoxic shock is harmful to the host as reflected by improved survival of mice lacking serotonin [6]. Platelet serotonin is moreover a crucial mediator of shock during immune complex-induced inflammation [8]. Platelet polyphosphate can exert both procoagulant and proinflammatory effects, influencing factor XII-mediated bradykinin release [7]. Furthermore, polyphosphate can directly increase vascular permeability [7]. As mentioned above, platelet ADP signaling has been shown to influence immune responses. In humans clopidogrel treat- 
Fig. 6. Lung hemorrhage. a-c Lung bleeding scores. a Control or Hps $3^{c o a}$ mice were infected with $K$. pneumoniae and sacrificed 12 or $40 \mathrm{~h}$ after infection. Mice were treated with PBS or clopidogrel (b) and control or $\mathrm{Hps} 3^{\mathrm{coa}}$ mice (c) were injected with antiGPIba antibody to deplete platelet counts to approximately $10 \%$, infected with $K$. pneumoniae, and sacrificed $40 \mathrm{~h}$ after infection. d-f Lung hemoglobin levels. d Control or $H p s 3^{\text {coa }}$ mice were infected with $K$. pneumoniae and sacrificed 12 or $40 \mathrm{~h}$ after infection. Mice were treated with PBS or clopidogrel (e) and control or Hps $3^{c o a}$ mice (f) were injected with anti-GPIba antibody to deplete platelet counts to approximately $10 \%$, infected with K. pneumoniae, and sacrificed $40 \mathrm{~h}$ after infection. g Representative images of lung bleeding (original magnification $\times 40$ ) of control or Hps $3^{\text {coa }}$ mice injected with anti-GPIba antibody to deplete platelet counts to approximately $10 \%$, infected with $K$. pneumoniae, and sacrificed $40 \mathrm{~h}$ after infection. Data are represented as scatter dot plots of 8 mice per group. Lines show the median. Differences between groups were not significant.

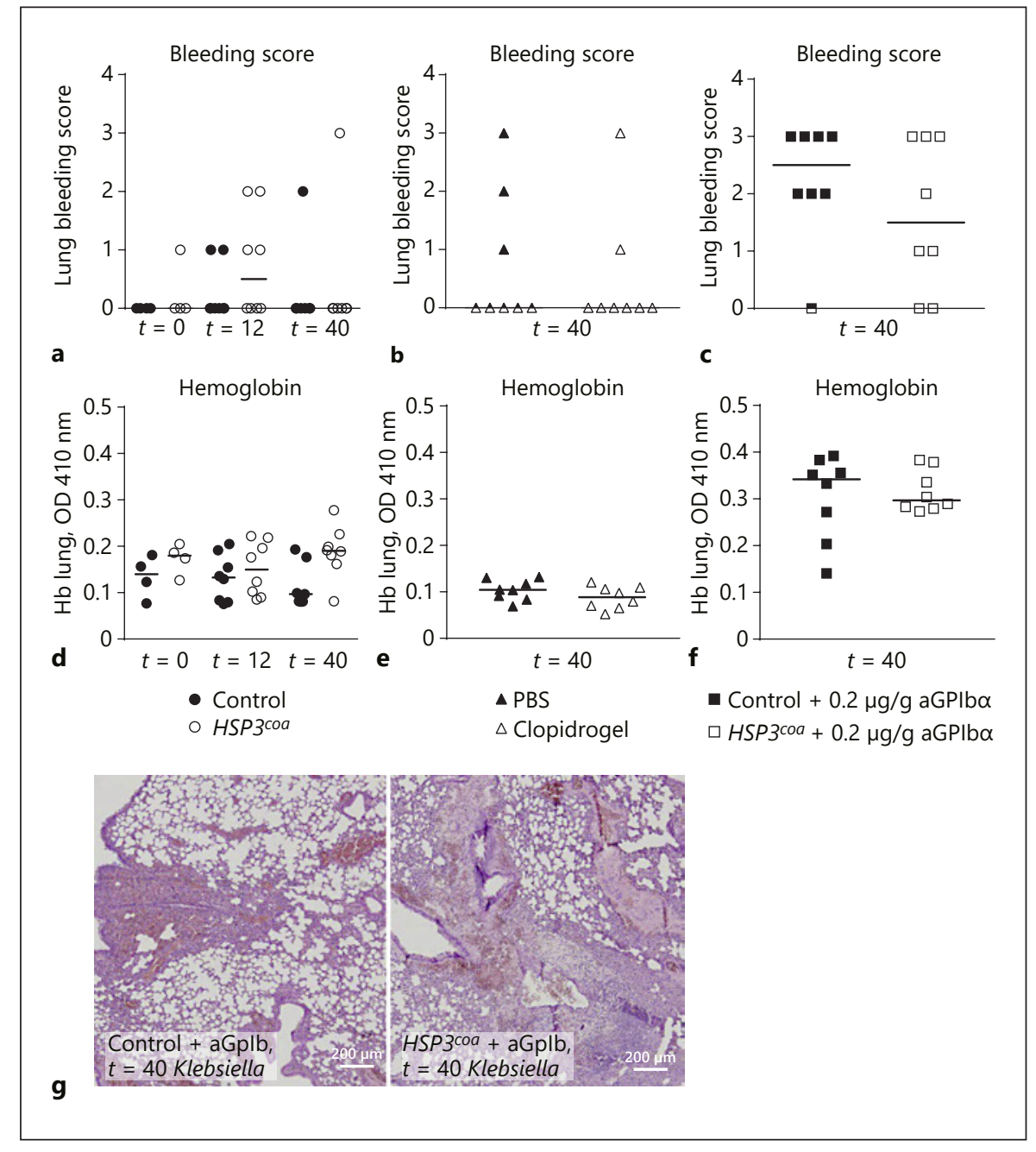

ment was associated with reduced platelet-leukocyte complex formation and cytokine levels $[5,26]$. In addition, in mice clopidogrel attenuated platelet activation ( $\mathrm{P}$ selectin expression), the formation of platelet-leukocyte aggregates, and reduced lung injury during polymicrobial abdominal sepsis induced by cecal ligation and puncture [30], underlining the importance of ADP in immune platelet function. In a murine atherosclerosis model, $\delta$ granule-deficient mice displayed reduced arterial inflammation, with fewer leukocytes and Mac-3-positive macrophages [24]. In our model of Gram-negative pneumonia and sepsis, however, $\delta$ granules and clopidogrel did not impact inflammation or host defense. In this respect it is important to note that unlike many other sepsis models, our model resembles the clinical scenario of sepsis originating from a primary site of infection (the lungs) with a steadily progressing inflammatory reaction as a result of a gradually increasing bacterial load.

Platelet-Dense Granules in Pneumonia and Sepsis
Platelet $\delta$ granules contributed to sepsis-induced thrombocytopenia, as indicated by a less severe decline in platelet counts in infected $H p s 3^{c o a}$ mice, especially in the setting of low pre-infection platelet counts. This effect is most likely mediated by reduced platelet activation, resulting in reduced consumption - as $\delta$ granules induce a positive feedback loop which increases platelet activation [1]. After low-dose PAR4AP stimulation ex vivo, $H p s 3^{\text {coa }}$ mice had reduced platelet activation as assessed by P-selectin expression, and in vivo reduced CXCL4 levels, but similar platelet P-selectin expression as controls. This might be explained by the fact that most activated platelets are attached to the vascular wall in vivo or already consumed, thereby restricting the ability to study these cells after blood withdrawal. Of note, while clopidogrel did not change thrombocytopenia during pneumosepsis, an earlier study reported a less severe decline in platelet counts in mice treated with clopidogrel during polymi- 


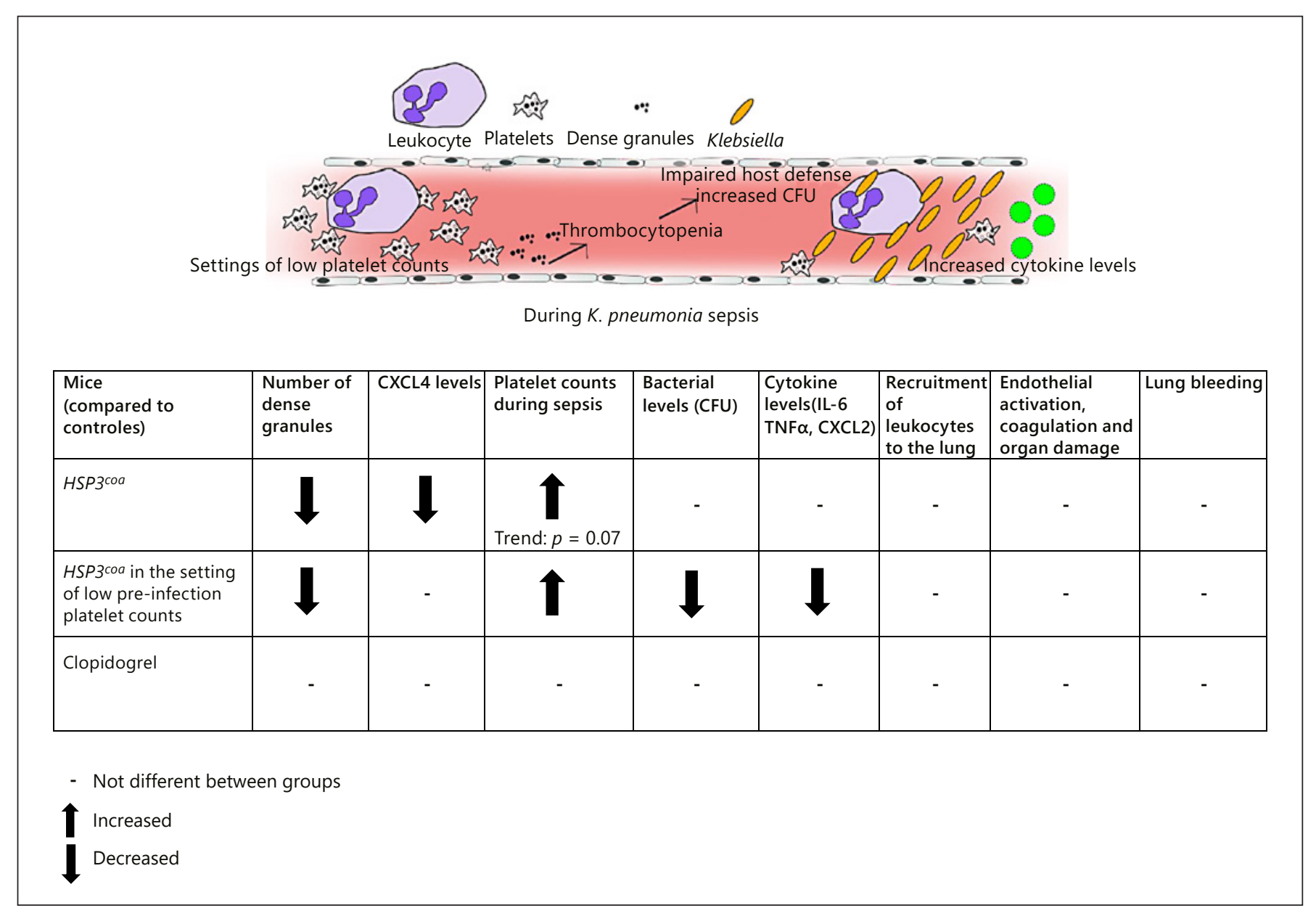

Fig. 7. Schematic overview of findings. In the setting of low pre-infection platelet counts, $\delta$ granule deficiency can result in decreased sepsis-induced thrombocytopenia. The result is higher platelet counts, which can aid in host defense against Klebsiella.

crobial abdominal sepsis [31], suggesting that platelet ADP signaling may be involved in sepsis-induced thrombocytopenia depending on the experimental model used. We have previously shown that at low platelet counts differences of $5 \%$ can already influence host defense in pneumonia-derived sepsis [19]. Indeed, whereas mice with platelet counts $<5 \times 10^{9} / \mathrm{L}$ demonstrated uncontrolled bacterial growth and macroscopic lung bleeding during pneumosepsis, in mice with platelet counts of $5-13 \times 10^{9} / \mathrm{L}$, host defense was partially restored and pulmonary hemorrhage partially prevented [19]. Therefore, we consider it likely that the lower lung bacterial loads seen in $\mathrm{Hps}^{\text {coa }}$ mice with low pre-infection platelet numbers are the consequence of the higher platelet counts still present in these mice at $40 \mathrm{~h}$ postinfection. The fact that clopidogrel did not modify sepsis-induced thrombocyto- penia indicates that the role of $\delta$ granules herein is not solely mediated via APD signaling or that ADP signaling was not completely abolished by clopidogrel treatment.

Platelets have been implicated in a variety of host responses during sepsis, including cytokine release, activation of coagulation, activation of the vascular endothelium $[9,14,19,32]$, and inflammatory responses. In addition, platelets can be involved in the occurrence of distant organ injury in sepsis $[9,14,32]$. The data presented here indicate that platelet $\delta$ granules or platelet ADP signaling have no role therein during Klebsiella pneumosepsis.

Platelets safeguard vascular integrity [19, 33]. The mechanisms by which platelets contribute to hemostasis in severe infection at least in part differ from those at play during sterile inflammation [12,33-35]. Previous studies have shown that platelet granule release protects against 
bleeding during thrombo-inflammatory brain infarction, but not during lipopolysaccharide-induced inflammation [36]. We have shown here that platelet $\delta$ granules are not involved in maintaining vascular integrity in the lungs during pneumosepsis. Even in the setting of preinfection thrombocytopenia, $\delta$ granules did not contribute to vascular integrity in spite of the fact that during late-stage infection $\mathrm{Hps} 3^{\text {coa }}$ mice had higher platelet counts relative to their littermate controls (and lower bacterial burdens).

We previously used this model of pneumonia-derived sepsis caused by the common human pathogen K. pneumoniae to study the role of platelets and platelet receptors in the host response to severe infection [11, 12, 19, 37]. This model is associated with a gradually growing bacterial load in the lungs with subsequent dissemination to distant body sites and organ injury, allowing studies on the role of components of innate immunity in early host defense as well as tissue damage during late-stage infection. We demonstrated that the number of platelets is a major denominator of their role in pneumosepsis [19], and identified platelet P-selectin [11] and GPVI [12] as mediators contributing to the control of bacterial growth. The current study suggests that platelet $\delta$ granules may contribute to thrombocytopenia in severe infection and can most likely thereby impair antibacterial defense during pneumonia-derived sepsis.

\section{Acknowledgements}

The authors would like to thank J.B. Daalhuisen, M. ten Brink, and R. de Beer (all Center for Experimental and Molecular Medicine, AMC, Amsterdam) for their technical support.

\section{Statement of Ethics}

All animal experiments were performed conforming to internationally accepted standards and the Institutional Animal Care and Use Committee of the Academic Medical Center approved all experiments.

\section{Disclosure Statement}

The authors have no conflicts of interest to declare.

\section{Funding Sources}

T.A.M.C. was funded by a grant from the Landsteiner Foundation for Blood Transfusion Research (LSBR grant 1351).

\section{Author Contributions}

T.A.M.C., A.F.d.V., C.v.t.V., and T.v.d.P. designed the study. T.A.M.C. acquired all the data. T.A.M.C., J.J.T.H.R., and O.J.d.B. analyzed the data. All authors were involved in the interpretation of the data. T.A.M.C. and T.v.d.P. drafted the manuscript and all authors reviewed and revised it critically for important intellectual content. All authors gave final approval for this version to be submitted.

\section{References}

1 Middleton EA, Weyrich AS, Zimmerman GA. Platelets in Pulmonary Immune Responses and Inflammatory Lung Diseases. Physiol Rev. 2016 Oct;96(4):1211-59.

2 Sharda A, Flaumenhaft R. The life cycle of platelet granules. F1000 Res. 2018 Feb;7:236.

3 Heijnen $\mathrm{H}$, van der Sluijs P. Platelet secretory behaviour: as diverse as the granules ... or not? J Thromb Haemost. 2015 Dec;13(12): 2141-51.

4 Jedlitschky G, Tirschmann K, Lubenow LE, Nieuwenhuis HK, Akkerman JW, Greinacher $\mathrm{A}$, et al. The nucleotide transporter MRP4 (ABCC4) is highly expressed in human platelets and present in dense granules, indicating a role in mediator storage. Blood. $2004 \mathrm{Dec}$; 104(12):3603-10.

5 Thomas MR, Storey RF. Effect of P2Y12 inhibitors on inflammation and immunity. Thromb Haemost. 2015 Aug;114(3):490-7.
6 Duerschmied D, Suidan GL, Demers M, Herr $\mathrm{N}$, Carbo C, Brill A, et al. Platelet serotonin promotes the recruitment of neutrophils to sites of acute inflammation in mice. Blood. 2013 Feb;121(6):1008-15.

7 Müller F, Mutch NJ, Schenk WA, Smith SA, Esterl L, Spronk HM, et al. Platelet polyphosphates are proinflammatory and procoagulant mediators in vivo. Cell. 2009 Dec;139(6): 1143-56.

8 Cloutier N, Allaeys I, Marcoux G, Machlus KR, Mailhot B, Zufferey A, et al. Platelets release pathogenic serotonin and return to circulation after immune complex-mediated sequestration. Proceedings of the National Academy of Sciences of the United States of America. 2018. doi: https://doi.org/10.1073/pnas.1720553115.
9 de Stoppelaar SF, van 't Veer C, van der Poll $\mathrm{T}$. The role of platelets in sepsis. Thromb Haemost. 2014 Oct;112(4):666-77.

10 Deppermann C, Kubes P. Platelets and infection. Semin Immunol. 2016 Dec;28(6):53645.

11 de Stoppelaar SF, Van't Veer C, Roelofs JJ, Claushuis TA, de Boer OJ, Tanck MW, et al. Platelet and endothelial cell P-selectin are required for host defense against Klebsiella pneumoniae-induced pneumosepsis. J Thromb Haemost. 2015 Jun;13(6):1128-38.

12 Claushuis TA, de Vos AF, Nieswandt B, Boon L, Roelofs J, de Boer OJ, et al. Platelet glycoprotein VI aids in local immunity during Gram-negative pneumonia-derived sepsis. Blood. 2018;131(8):864-76. 
13 Rossaint J, Kühne K, Skupski J, Van Aken H, Looney MR, Hidalgo A, et al. Directed transport of neutrophil-derived extracellular vesicles enables platelet-mediated innate immune response. Nat Commun. 2016 Nov;7:13464.

14 Wong CH, Jenne CN, Petri B, Chrobok NL, Kubes P. Nucleation of platelets with bloodborne pathogens on Kupffer cells precedes other innate immunity and contributes to bacterial clearance. Nat Immunol. 2013 Aug. 14(8):785-92.

15 Deppermann C, Kubes P. Start a fire, kill the bug: the role of platelets in inflammation and infection. Innate Immun. 2018 Aug;24(6): $335-48$.

16 Kapur R, Semple JW. Platelets as immunesensing cells. Blood Adv. 2016 Nov; 1(1):10-4.

17 Kapur R, Semple JW. The nonhemostatic immune functions of platelets. Semin Hematol. 2016 Apr;53 Suppl 1:S2-6.

18 Kapur R, Zufferey A, Boilard E, Semple JW Nouvelle cuisine: platelets served with inflammation. J Immunol. 2015 Jun;194(12): 5579-87.

19 de Stoppelaar SF, van't Veer C, Claushuis TA, Albersen BJ, Roelofs JJ, van der Poll T. Thrombocytopenia impairs host defense in Gram-negative pneumonia-derived sepsis in mice. Blood. 2014 Dec;124(25):3781-90.

20 Kang CI, Song JH, Chung DR, Peck KR, Ko KS, Yeom JS, et al.; Korean Network for Study of Infectious Diseases (KONSID). Risk factors and pathogenic significance of severe sepsis and septic shock in 2286 patients with gram-negative bacteremia. J Infect. 2011 Jan; 62(1):26-33.

21 Paczosa MK, Mecsas J. Klebsiella pneumoniae: Going on the Offense with a Strong Defense. Microbiol Mol Biol Rev. 2016 Jun 80(3):629-61.
22 Novak EK, Sweet HO, Prochazka M, Parentis $\mathrm{M}$, Soble R, Reddington M, et al. Cocoa: a new mouse model for platelet storage pool deficiency. Br J Haematol. 1988 Jul;69(3):371-8.

23 Swank RT, Novak EK, McGarry MP, Rusiniak ME, Feng L. Mouse models of Hermansky Pudlak syndrome: a review. Pigment Cell Res. 1998 Apr;11(2):60-80.

24 King SM, McNamee RA, Houng AK, Patel R, Brands M, Reed GL. Platelet dense-granule secretion plays a critical role in thrombosis and subsequent vascular remodeling in atherosclerotic mice. Circulation. 2009 Sep; 120(9):785-91.

25 Nishibori M, Cham B, McNicol A, Shalev A, Jain N, Gerrard JM. The protein CD63 is in platelet dense granules, is deficient in a patient with Hermansky-Pudlak syndrome, and appears identical to granulophysin. J Clin Invest. $1993 \mathrm{Apr} ; 91(4): 1775-82$.

26 Thomas MR, Outteridge SN, Ajjan RA, Phoenix F, Sangha GK, Faulkner RE, et al. Platelet P2Y12 Inhibitors Reduce Systemic Inflammation and Its Prothrombotic Effects in an Experimental Human Model. Arterioscler Thromb Vasc Biol. 2015 Dec;35(12):2562-70.

27 Herbert JM, Savi P. P2Y12, a new platelet ADP receptor, target of clopidogrel. Semin Vasc Med. 2003 May;3(2):113-22.

28 Morowski M, Vögtle T, Kraft P, Kleinschnitz C, Stoll G, Nieswandt B. Only severe thrombocytopenia results in bleeding and defective thrombus formation in mice. Blood. 2013 Jun;121(24):4938-47.

29 Levi M, Schultz M. Coagulopathy and platelet disorders in critically ill patients. Minerva Anestesiol. 2010 Oct;76(10):851-9.
30 Liverani E, Rico MC, Tsygankov AY, Kilpatrick LE, Kunapuli SP. P2Y12 Receptor Modulates Sepsis-Induced Inflammation. Arterioscler Thromb Vasc Biol. 2016 May;36(5):96171.

31 Seidel M, Winning J, Claus RA, Bauer M, Lösche W. Beneficial effect of clopidogrel in a mouse model of polymicrobial sepsis. J Thromb Haemost. 2009 Jun;7(6):1030-2.

32 Xiang B, Zhang G, Guo L, Li XA, Morris AJ, Daugherty A, et al. Platelets protect from septic shock by inhibiting macrophage-dependent inflammation via the cyclooxygenase 1 signalling pathway. Nat Commun. 2013;4(1) 2657.

33 Boulaftali Y, Hess PR, Getz TM, Cholka A, Stolla M, Mackman N, et al. Platelet ITAM signaling is critical for vascular integrity in inflammation. J Clin Invest. $2013 \mathrm{Feb} ; 123(2)$ : 908-16.

34 Goerge T, Ho-Tin-Noe B, Carbo C, Benarafa C, Remold-O'Donnell E, Zhao BQ, et al. Inflammation induces hemorrhage in thrombocytopenia. Blood. 2008 May;111(10):495864.

35 Gros A, Syvannarath V, Lamrani L, Ollivier V, Loyau S, Goerge T, et al. Single platelets seal neutrophil-induced vascular breaches via GPVI during immune-complex-mediated inflammation in mice. Blood. 2015 Aug; 126(8): 1017-26.

36 Deppermann C, Kraft P, Volz J, Schuhmann MK, Beck S, Wolf K, et al. Platelet secretion is crucial to prevent bleeding in the ischemic brain but not in the inflamed skin or lung in mice. Blood. 2017 Mar;129(12):1702-6.

37 de Stoppelaar SF, Claushuis TA, Jansen MP, Hou B, Roelofs JJ, van 't Veer C, et al. The role of platelet MyD88 in host response during gram-negative sepsis. J Thromb Haemost. 2015 Sep;13(9):1709-20. 